\title{
Endostar Plus Apatinib Successfully Achieved Long Term Progression-Free Survival in Refractory Ovarian Cancer: A Case Report and Literature Review
}

\section{Chunmei Xiao* \\ Fangye $\mathrm{Xu}^{*}$ \\ Rong Wang \\ Qi Liang \\ Kai Shen \\ Jiali Xu \\ Lianke Liu (D)}

Department of Oncology, The First Affiliated Hospital of Nanjing Medical University, Nanjing, 210029, People's Republic of China

*These authors contributed equally to this work
Correspondence: Lianke Liu; Jiali Xu Department of Oncology, The First Affiliated Hospital of Nanjing Medical University, Nanjing, 210029, People's

Republic of China

$\mathrm{Tel}+86 \quad 1395 \quad 1623290$

Fax +8625-837I-0040

Email liulianke@jsph.org.cn; xujiali@jsph. org.cn
Background: Ovarian cancer (OC) is a common malignancy in the gynecological tumor. Standard treatment for ovarian cancer is surgery and chemotherapy based on paclitaxel and platinum. However, traditional chemotherapy for ovarian cancer is limited by drug resistance and systemic side effects. It is imperative to explore effective treatment options for refractory ovarian cancer.

Case Presentation: A 52-year-old female initially presented with lower abdominal distension and migratory pain. After the laparoscopic exploration and biopsy, immunohistochemistry showed poorly differentiated adenocarcinoma originated from ovarian (cT3NxM1, stage IV, peritoneal and abdominal wall metastasis). The next generation sequence detected ERRFI1 (T187A, exon4) mutation.

Results: The patient received first-line chemotherapy (paclitaxel, nedaplatin plus avastin), followed by maintenance therapy with gefitinib, achieving a 15-month progression-free survival (PFS). After disease progression and second-line treatment failure, endostar plus apatinib was administered for 14 cycles and she obtained a PFS of 14 months without longterm adverse events.

Conclusion: We believe that the ERRFI1 gene may be a potential target of gefitinib. Importantly, endostar combined with apatinib is worth recommending for maintenance treatment in refractory ovarian cancer.

Keywords: ovarian cancer, antiangiogenic therapy, endostar, apatinib, gefitinib

\section{Background}

Ovarian cancer is one of the most common malignant tumors in gynecology. In 2018 statistics indicate a burden of 225, 000 new cases and 140,200 deaths. ${ }^{1}$ Due to the lack of early symptoms and effective screening strategies, approximately $70 \%$ of patients with ovarian cancer are usually diagnosed at a late stage, not to mention the even higher mortality. ${ }^{2}$ According to previous studies, the 10 -year progressionfree survival (PFS) rate of patients with recurrent disease is only $15 \%$, and the median overall survival (OS) is $12-24$ months. ${ }^{3}$ Standard treatment for ovarian cancer is surgery and chemotherapy based on paclitaxel and platinum. However, traditional chemotherapy for ovarian cancer is limited by drug resistance and systemic side-effects. Therefore, joint efforts are necessary to develop novel therapies for chemotherapy resistance and recurrent ovarian cancer, For patients with 
BRCA mutations, maintenance therapy with approved PARP inhibitors can significantly improve PFS. ${ }^{4}$ Bevacizumab combined with platinum-based dual therapy, and PARP inhibitors combined with anti-angiogenesis drugs or immune checkpoint suppression are also under active research. ${ }^{5}$ Based on the patient's genes and tumor molecular characteristics, targeted therapy allows for an individualized approach in the management of ovarian cancer. Both anti-angiogenic therapies alone and combined with chemotherapy have shown efficacy in ovarian cancer. ${ }^{6-8}$ Here, we report an ovarian cancer patient who achieved partial response (PR) from antiangiogenic therapy with a PFS of 14 months after multiple-lines failure, and combine with a literature review to demonstrate the effectiveness of this novel combined regimen in ovarian cancer.

\section{Case Presentation}

A 52-year-old female was admitted to the hospital in January 29, 2018, initially presenting with lower abdominal distension and migratory pain. Her family history was unremarkable and she is a non-smoker with unknown allergic history. The Eastern Cooperative Oncology Group score was one point. The serum antigen-125 (CA125) was $326.4 \mathrm{U} / \mathrm{mL}$, and the abdominal enhanced $\mathrm{CT}$ (Figure 1A) showed an enlarged uterus, irregular soft tissue-like mass on the left posterior, and multiple nodules and masses in the peritoneum and omentum on both sides. Laparoscopic biopsy was performed and the histopathology confirmed poorly differentiated adenocarcinoma originating from the ovary (Figure 2). Immunohistochemical analysis of omentum tissue was positive for Pax2, PaX8, CK7, WT-1, ER, P53. According to pathology and immunohistochemistry, the clinical diagnosis was advanced ovarian cancer (FIGO stage IV, cT3NxM1). In April 2018, NGS detected ERRFI1 (T187A, exon4) mutation, but no "actionable" drugs have been approved by FDA (Table 1).

The patient received 6 cycles of paclitaxel, nidaplatin and avastin (intravenous paclitaxel $180 \mathrm{mg} / \mathrm{m}^{2} \mathrm{~d} 1$; nidaplatin $50 \mathrm{mg} / \mathrm{m}^{2} \mathrm{~d} 1-\mathrm{d} 2$; avastin $300 \mathrm{mg} / \mathrm{m}^{2} \mathrm{~d} 1$ ) every 21 day cycle starting in mid-March 2018. According to the literature, we found ERRFI1 is a regulatory factor in the EGFR pathway, and it has been reported that cases treated with gefitinib have a higher remission rate. Therefore, the patient began to receive oral targeted therapy of gefitinib 250mg qod combined with chemotherapy in May 2018. After 4 courses, a CT scan revealed a significant reduction of pelvic mass (Figure 1B) and achieved partial remission (SD) according to RECIST 1.1. Considering that the patient cannot continue to afford avastin, medical insurance cannot be reimbursed in our country. Since then, gefitinib monotherapy was given for ten months. CT scan was performed on May 27, 2019, and the treatment evaluation was PD. Currently, no data shows the survival benefit of secondary cell reduction. Considering the high risk of complications after cytoreductive surgery, the patient with peritoneal metastasis and splenic metastasis refused the second operation. Subsequently, the patient received a new chemotherapy regimen in September 2019 with docetaxel $\left(90 \mathrm{mg} / \mathrm{m}^{2} \mathrm{~d} 1\right)$ plus carboplatin $\left(400 \mathrm{mg} / \mathrm{m}^{2} \mathrm{~d} 1\right)$. However, the CT scan proved PD again and the therapy was unsuccessful (Figure 1C). AntiVEGF therapy has been widely proven to be effective in the treatment of various malignant tumors in recent years. Since the patient has received multiple lines of chemotherapy, the patient could not tolerate the side-effects of chemotherapy and the inconvenience of chemotherapy administration. After full communication, the patient received a novel regimen with antiangiogenic drug endostar (30 mg, civ d1-4) plus apatinib (250 mg, po qod) from November 18, 2019. In consideration of the off-label use of endostar and apatinib, we communicated adequately with the patient before signing the off-label consent. Response evaluations after every two months during 10 cycles of treatment showed stable disease SD (Figure 1D). On December 2020, CT scan revealed that the mass on the right side was larger than before and accompanied by peritoneal metastasis, and simultaneously the CA-125 increased significantly. In view of the PFS of 13 months of this regimen, we advised the patient to continue endostar plus apatinib as maintenance therapy although the results showed a slight PD. However, her serum CA-125 level rose again one month and the therapeutic evaluation was PD. Generally speaking, PFS for apatinib combined with endostar therapy was 14 months. The patient was followed up for about 30 months and she is still alive now. During the treatment, CT and CA-125 (Figure 3) were reviewed every 2 months. The major adverse events observed were grade 1 hand-foot syndrome (HFS) and grade 2 gastrointestinal reactions. No hypertension or proteinuria occurred.

The patient and his family provided written informed consent for publication of this report and accompanying images. We confirmed with the institutional review board of the First Affiliated Hospital of Nanjing Medical 

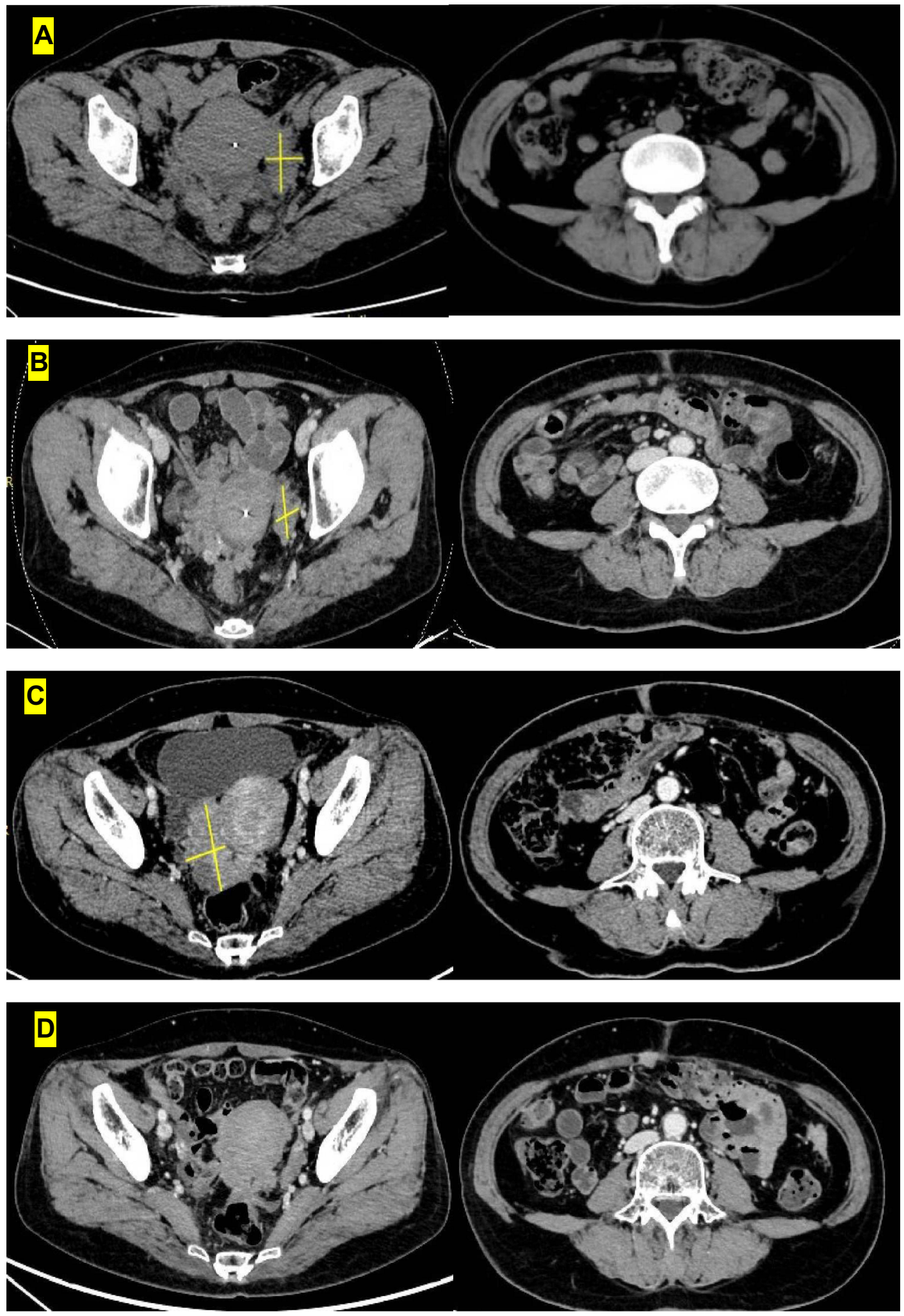

Figure I Changes on CT scans during treatment.

Notes: (A) Left posterior lesion of uterus $(22.2 \mathrm{~mm} / 39.7 \mathrm{~mm})$ and Peritoneal metastasis $(7.7 \mathrm{~mm})$ on February 19, 2018; (B) Left posterior lesion of uterus (19.5mm/ $32.1 \mathrm{~mm}$ ) and Peritoneal metastasis $(5.4 \mathrm{~mm})$ on August 27, 2018; Changes on CT scans before and after endostar combined with apatinib treatment. (C) Left posterior lesion of uterus $(42.2 \mathrm{~mm} / 36.6 \mathrm{~mm})$ and Peritoneal metastasis $(11.1 \mathrm{~mm})$ on October 28, 2019; (D) Left posterior lesion of uterus $(23.4 \mathrm{~mm} / 19.0 \mathrm{~mm})$ and Peritoneal metastasis $(9.7 \mathrm{~mm})$ on August 31, 2020.

Abbreviation: $\mathrm{CT}$, computed tomography. 


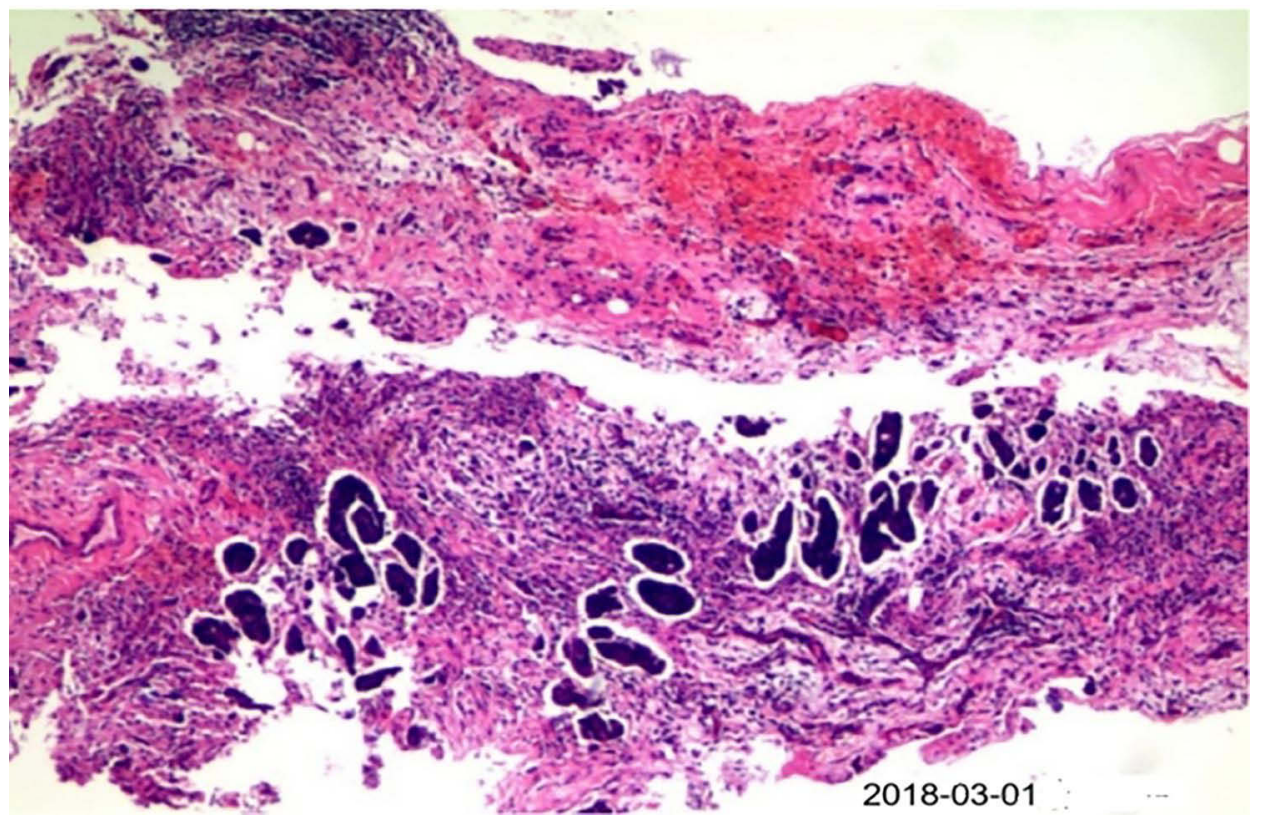

Figure $2 \mathrm{H} \& \mathrm{E}$ staining with surgical specimens of the patient.

University that institutional approval was not required to publish this case report.

\section{Discussion}

In recent decades, the incidence and mortality of ovarian cancer in China have been increasing rapidly. ${ }^{9,10}$ Despite numerous improvements in surgery, chemotherapy, and postoperative adjuvant chemotherapy, only about $30 \%$ of platinum-sensitive relapsed patients respond to second-line chemotherapy. Approximately $75 \%$ of women with advanced-stage ovarian cancer will relapse and die, and the 5 -year overall survival rate is only $40-50 \% .{ }^{11}$ In view of the widespread adoption of primary surgical cytopenias, secondary cytoreductive surgery is also considered for patients with recurrent disease. In most cases, repetitive or secondary cytoreductive surgery for recurrent ovarian cancer refers to surgery performed within a certain period of time after the completion of primary treatment (the diseasefree interval exceeds 6 to 12 months), with the goal of reducing tumors. Many single-institution and multiinstitution retrospective reviews and meta-analysis support the procedure. ${ }^{12}$ However, in a trial of platinum-sensitive patients with recurrent ovarian cancer, chemotherapy after the second surgery for cell reduction did not lead to a longer overall survival than chemotherapy alone. ${ }^{13}$ Therefore, whether to choose surgery is based on the patient's age, general health, and patient's wishes. The individualized decision is to carefully weigh the potential benefits. Most ovarian cancers are sporadic, and about $15 \%$ of cases are considered to be genetically related to BRCA mutations. ${ }^{14}$ Latest studies have shown that BRCA1 patients have an increased risk of morbidity after age 40 , while BRCA2 patients have an increased risk of morbidity after age $50 .^{15}$ Since the discovery of BRCA in 1990, more and more researchers have realized the vital role of BRCA in ovarian tumorigenesis and applied it as a therapeutic target and a potential screening method. Germline mutations of BRCA1 and BRCA2 genes, which encode proteins necessary for double-strand DNA repair, break through homologous recombination and lead to increased cancer susceptibility. ${ }^{16}$ BRCA1 can develop resistance to platinumbased chemotherapy through involvement in the FA/

Table I DNA Sequencing Tumor Tissue Genetic Variation, 450 Gene DNA Sequencing Detected I Tumor-Related Gene Mutation of I Gene

\begin{tabular}{|l|l|l|l|}
\hline Gene Name & Mutation Information & Tumor Mutation Burden & Microsatellite Instability Results \\
\hline ERRFII & TI87A exon4 & 3.2 Muts/Mb (<75\%) & MSS \\
\hline
\end{tabular}

Note: Variation form: Threonine at position 187 of the ERRFII gene is changed to alanine. 


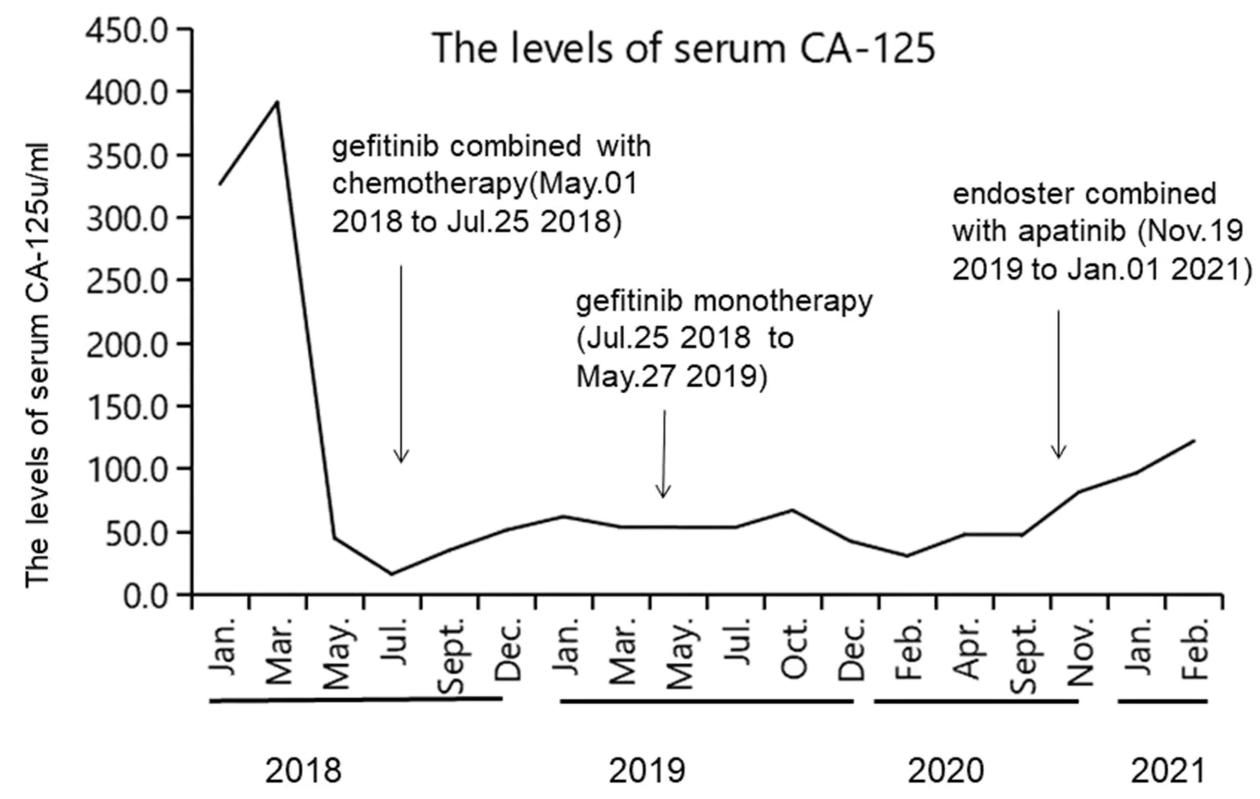

Figure 3 CA- 125 levels change during treatment. After gefitinib maintenance treatment started in May 2018 , the level of CA- 125 remained stable at about $50 \mathrm{U} / \mathrm{mL}$ In October 2019, the CA- 125 increased to $67.2 \mathrm{U} / \mathrm{mL}$. Endostar combined with apatinib treatment started in November 2019, and the CA-125 level was maintained within 50 $\mathrm{U} / \mathrm{mL}$ again and entered a more stable state.

BRCA1, HR, and NER repair pathways. ${ }^{17}$ Treatment after first-line therapy in EOC depends on the platinum-free interval (PFI) and the side-effects of the previous therapy. Unfortunately, the results of BRCA1/2 carriers challenge the traditional definition of platinum resistance. Researches have confirmed that patients with BRCA mutations have a better response to platinum therapy. ${ }^{18-20}$ Recently, BRCA1/2 mutation screening has become routine examination in clinical practice, becoming one of the few successful clinical interventions for ovarian cancer. In 2005, two research groups respectively discovered that PARP inhibition induces synthetic lethality of mutant BRCA1 or BRCA2 cancer. $^{21}$ In 2009, the first trial of PARP inhibitors (PARPi) was published in solid tumors patients with BRCA mutations. $^{22}$ Currently, multiple PARPi have been approved by the FDA for advanced ovarian cancer and/or breast cancer, such as olaparib in 2014, rucaparib in 2016, niraparib in 2017, and talazoparib in $2018 .^{23}$ As molecularly targeted agents emerging, there are more options for the treatment of refractory ovarian cancer. Through genetic testing or circulating ctDNA, mutant genes can be effectively targeted for treatment. Our patient has no BRCA or other FDA-approved targeted mutations. The patient's NGS detected ERRFI1 mutation (the product of mitogeninducible gene 6), located on the chromosome 1. Through the TCGA database, we learned the expression and survival curve of this gene in ovarian cancer (Figure 4). ERRFI1 is docked with the growth factor receptor (EGFR) kinase domain through its ERBB binding region, ${ }^{24}$ inhibiting EGFR activation and downstream signal transduction. As ERRFI1 was identified as a feedback inhibitor of EGFR, ${ }^{25}$ a high level of ERRFI1 can increase the resistance of cancer cells to EGFR tyrosine kinases (TKIs) and limit the therapeutic effects of EGFR TKIs, such as gefitinib or erlotinib. Gefitinib is an oral, competitive inhibitor of the EGFR TKI, also known as HER1 or ErbB-1, which can block EGFR mutations signal and overactive target cancer cell metastasis. $^{26}$ Several clinical studies have shown the obvious superiority of gefitinib in NSCLC patients with EGFR mutations for significant improvement in PFS and low incidence of adverse events. Interestingly, its effects are generally reversible. ${ }^{27,28}$ Two cases have been reported, one with a low ERRFI1/EGFR ratio and the other one with ERRFI1 gene function-loss mutation. Gefitinib and erlotinib were respectively given and both patients achieved a higher remission rate. ${ }^{29,30}$ In our case, the patient with mutation site ERRFI1 received conventional first-line chemotherapy combined with gefitinib maintenance treatment, achieving PFS for 15 months without obvious adverse reactions. These clinical cases suggest that the EEFI1 gene may be a potential cancer target of gefitinib.

Due to the lack of standardized therapy, treatment for refractory ovarian cancer is individualized based on patients' characteristics and previous treatment. 

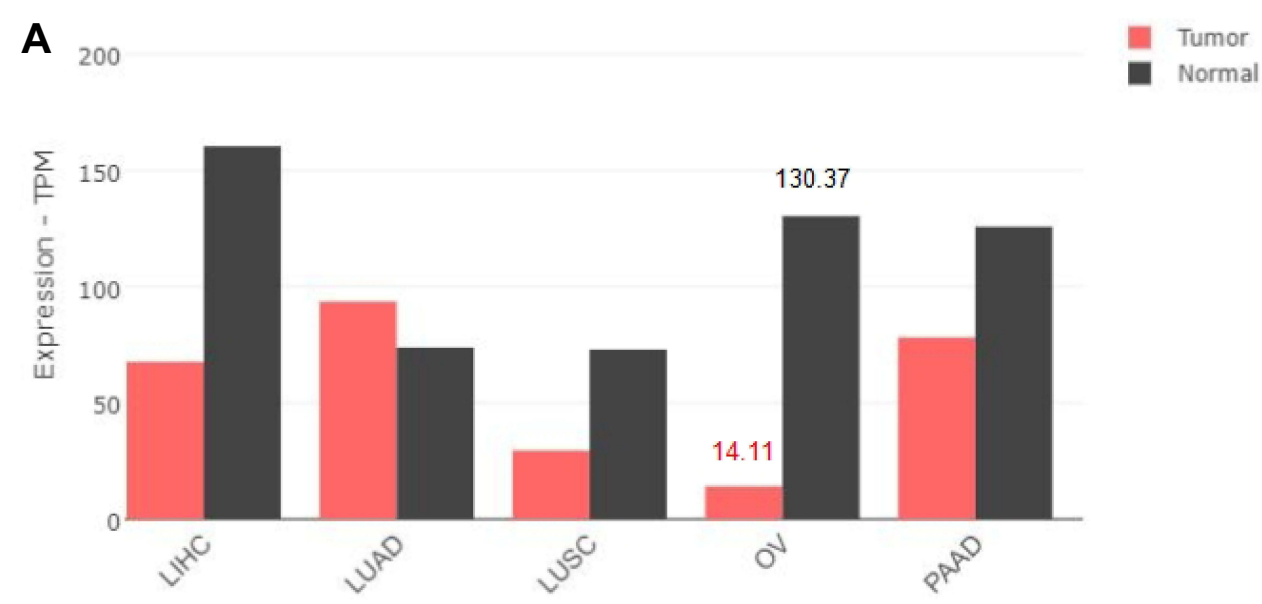

B Overall Survival

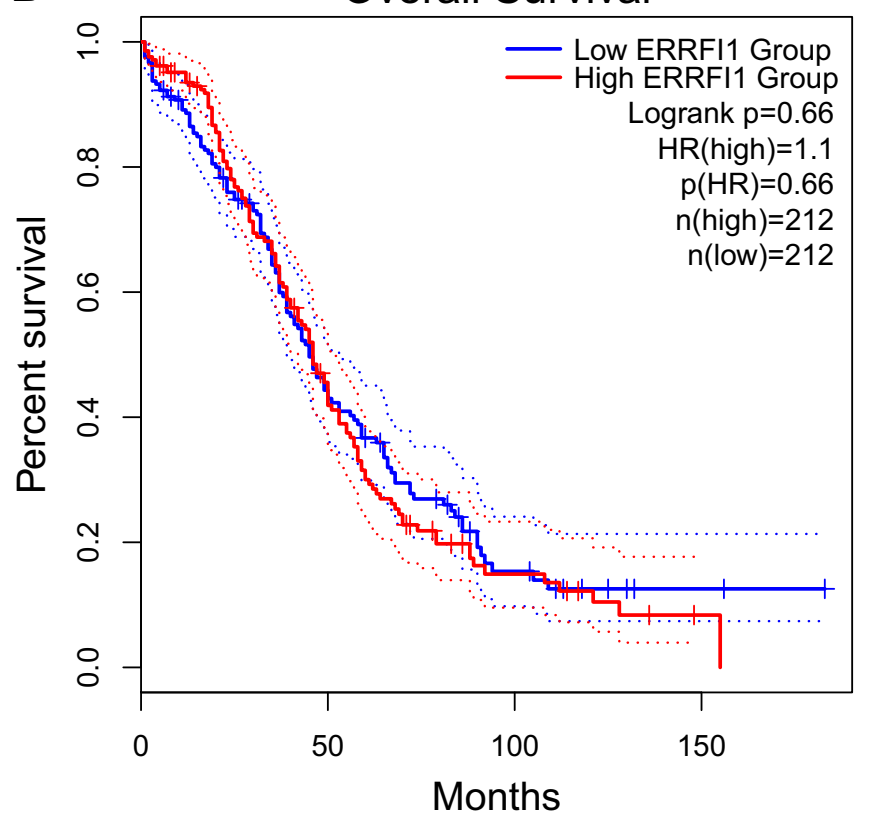

Figure 4 (A) The gene expression profile across some tumor samples and paired normal tissues. The height of bar represents the median expression of certain tumor type or normal tissue. (B) Expression of ERRFII and Survival Curve of Ovarian Cancer.

Abbreviations: LIHC, liver hepatocellular carcinoma; LUAD, lung adenocarcinoma; LUSC, lung squamous cell carcinoma; OV, ovarian serous cystadenocarcinoma; PAAD, pancreatic adenocarcinoma.

Increasing treatment options are emerging, including vascular endothelial growth factors (VEGF) inhibitors and TKI, such as bevacizumab, pazopanib, and nintedanib. Therefore, it is crucial to choose an appropriate treatment regimen. Up to now, endostar combined with apatinib has not been reported in EOC and both of them are new biological products for angiogenesis inhibition. Angiogenesis is a key factor in the growth of malignant tumors, and sustained angiogenesis is closely associated with tumor occurrence, development, and metastasis. ${ }^{31}$ Studies have confirmed that anti-angiogenesis drugs have been beneficial for the treatment of many ovarian cancer patients who have failed in multi-line chemotherapy. Bevacizumab, one of the most studied target therapies, has been approved for first-line and second-line treatment of advanced EOC. ${ }^{32}$ In addition, researches on apatinib and endostar has also been advancing. Bevacizumab mainly neutralizes VEGF-A and prevents binding to VEGFR-1, 2 to achieve angiogenesis inhibition while both apatinib and endostar not only are selective inhibitors of VEGFR-2 but also retain other anti-angiogenic activities. These three drugs have shown inhibitory effects on angiogenesis and tumors in the zebrafish model. Importantly, the effects of endostar and apatinib are 
superior to bevacizumab. ${ }^{33}$ Single antiangiogenic agents are prone to drug resistance and tumor recurrence. Jain published a paper in Science and proposed that the ideal tumor treatment requires the "cocktail therapy" of antiangiogenesis therapy to normalize blood vessels. ${ }^{34}$ Therefore, choosing a multi-target anti-angiogenic agent or a combination of anti-angiogenic agents may circumvent the anti-angiogenic drug resistance alone and obtain clinical benefit. An experimental study found that the combination of recombinant human endostatin and bevacizumab has an additive effect. ${ }^{35}$ Our clinical team has previously achieved benefits in the treatment of a Merkel cell carcinoma patient who cannot tolerate chemotherapy and radiotherapy. The patient achieved partial remission after 2 months of treatment with endostar 30mg civ24h d14 plus apatinib $250 \mathrm{mg}$ qod PO, and achieved 6.5 months PFS with tolerable adverse reactions. ${ }^{36}$ Thus, we selected an individualized treatment regimen with endostar plus apatinib for the patient in this case.

Apatinib, approved and marketed in China in 2014, is an oral new type of TKI. It could highly selectively compete for the ATP-binding site of intracellular VEGFR-2 and block its downstream signal transduction, thereby playing an antitumor part via robust inhibition of tumor angiogenesis. ${ }^{37,38}$ In addition, apatinib can reverse multidrug resistance (MDR) of tumor cells mediated by multidrug transporter protein $\mathrm{ABCB} 1$ (p-glycoprotein) via suppressing its activity in pumping out chemotherapy drugs to improve chemotherapy efficacy. ${ }^{7,39}$ Several cases showed that apatinib therapy significantly improves OS and PFS with acceptable safety although patients have previously received various chemotherapy regimens. ${ }^{40}$ Apatinib may be a feasible option for recurrent platinumresistant EOC and has good efficacy and controllable toxicity. ${ }^{41,42}$ Further, apatinib is cheaper and easier to administer compared with bevacizumab. ${ }^{43}$ According to the research of Scott et al, apatinib could reverse the P-glycoprotein ABCB1-and ABCG2-mediated multidrug resistance in drug-resistant solid tumor cells by inhibiting their transport function, to circumvents cancer cell resistance to other anti-tumor drugs. ${ }^{44}$ Endostar is an anti-tumor angiogenesis drug developed in China and was approved by the China National Food and Drug Administration (CFDA) for NSCLC in September 2005. It's a modified human endostatin retaining endogenous endostatin function. Studies demonstrate endostar can not only down-regulate the expression of VEGFs and VEGF receptors (VEGFRs) but also inhibit the activation of ERK, p38 MAPK, and
$\mathrm{Akt}^{45}$ which also up-regulate the level of SRCIN1 protein in vascular endothelial cells and affect the Src signaling pathway and restore blood vessel normalization. ${ }^{46,47}$ Compared with anti-angiogenesis targeted therapies such as monoclonal antibodies and TKIs, endostar can regulate multiple signaling pathways, widely targeting signaling molecules, growth factors, and enzymes. Moreover, endostatin specifically binds to nucleolar proteins on the cell surface and inhibits tumor lymphangiogenesis and lymphatic metastasis by down-regulating VEGF-C levels and VEGFR-3 gene expression. ${ }^{48}$ A large number of clinical data and experience have been obtained in the research of applying endostar to treat different cancers. ${ }^{46}$ In recent years, endostar combined with chemotherapy has been effective in the treatment of ovarian cancer, and significantly improve PFS of ovarian cancer with the recurrent epithelial resistance of platinum. ${ }^{46}$ Studies have analyzed that endostar can block the metastasis, invasion, and angiogenesis of ovarian cancer cells by inhibiting the activation of PD-L1 and STAT3. ${ }^{49}$ Our patient, treated with endostar (30mg civ24h d1-4) plus apatinib (250mg qod PO) for 14 months, achieved stable disease with good safety, controllable adverse reactions, and no long-term adverse events. The recommended dosage of endostar's instructions is $7.5 \mathrm{mg}$ and administrated by intermittent intravenous (IIV) infusion for 3-4 h per day during a 14-day period. Since 2010, the method of continuous intravenous (CIV) infusion via an infusion pump to increase the dose for short-term maintenance has been introduced and widely off-label used in clinical practice in China. This method is safe and effective and it can reduce toxicity, extend the retention time in the blood, and increase the active ingredient in the target tissue. Besides, it can improve the medical compliance and life treatment capacity of patients. ${ }^{50}$ Hansma et al have studied the safety of $\mathrm{CI}$ at different doses and indicated that this method of administration is safe. ${ }^{51}$ Chen et al have shown that there is a linear correlation between the exposure of the body to endostar and the administered dose between 7.5 and $30 \mathrm{mg} / \mathrm{m}^{2} / \mathrm{day}^{52}$ Secondly, due to the small body surface area of our patient, and HFS was unbearable during the initial daily treatment with apatinib. Considering the study of $\mathrm{Hu}$ et al, the recommended dose was $500 \mathrm{mg}$ daily and the rate of grade $3 / 4$ toxicity was significantly decreased, whose efficacy was similar to that of the daily regimen of $850 \mathrm{mg}$ in breast carcinoma. ${ }^{53} \mathrm{In}$ our case, we used apatinib at an initial dose of $250 \mathrm{mg} / \mathrm{d}$, lower than the amount of apatinib applied in previous 
clinical trials. Thus, common side effects such as high blood pressure and proteinuria were avoided.

In view of the clinical effectiveness of endostar plus apatinib and anti-angiogenesis treatment experience, endostar combined with apatinib might be a therapeutic option in refractory ovarian cancer to prolong survival. We have confirmed that the combination of two anti-angiogenic agents therapy is feasible in refractory ovarian cancer. Further studies and clinical data are needed to prove ovarian tumors with visceral metastasis or other cancers.

\section{Conclusion}

In conclusion, treatment for refractory ovarian cancer should be individualized. Our case indicated that the ERRFI1 gene may be a potential target of gefitinib and endostar plus apatinib is worth recommending for the maintenance treatment in refractory ovarian cancer. In addition, we confirmed that anti-angiogenic therapy is feasible in refractory ovarian cancer.

\section{Acknowledgments}

The authors thank the patient and his family members for their participation in this study and for their agreement to the publication of the report.

\section{Author Contributions}

All authors made a significant contribution to the work reported, whether that is in the conception, study design, execution, acquisition of data, analysis and interpretation, or in all these areas; took part in drafting, revising or critically reviewing the article; gave final approval of the version to be published; have agreed on the journal to which the article has been submitted; and agree to be accountable for all aspects of the work.

\section{Disclosure}

The authors report no conflicts of interest in this work.

\section{References}

1. Bray F, Ferlay J, Soerjomataram I, Siegel RL, Torre LA, Jemal A. Global cancer statistics 2018: GLOBOCAN estimates of incidence and mortality worldwide for 36 cancers in 185 countries. CA Cancer J Clin. 2018;68(6):394-424. doi:10.3322/caac.21492

2. Aravantinos G, Pectasides D. Bevacizumab in combination with chemotherapy for the treatment of advanced ovarian cancer: a systematic review. J Ovarian Res. 2014;7:57. doi:10.1186/1757-2215-7-57

3. Corrado G, Salutari V, Palluzzi E, Distefano MG, Scambia G, Ferrandina G. Optimizing treatment in recurrent epithelial ovarian cancer. Expert Rev Anticancer Ther. 2017;17(12):1147-1158. doi: $10.1080 / 14737140.2017 .1398088$
4. Barrena Medel NI, Wright JD, Herzog TJ. Targeted therapies in epithelial ovarian cancer. J Oncol. 2010;2010:314326. doi:10.1155/ 2010/314326

5. Pignata S, Cecere SC, Du Bois A, Harter P, Heitz F. Treatment of recurrent ovarian cancer. Ann Oncol. 2017;28(suppl_8):viii51-viii56. doi:10.1093/annonc/mdx441

6. He M, Wei MJ. Reversing multidrug resistance by tyrosine kinase inhibitors. Chin $J$ Cancer. 2012;31(3):126-133. doi:10.5732/ cjc.011.10315

7. Mi YJ, Liang YJ, Huang HB, et al. Apatinib (YN968D1) reverses multidrug resistance by inhibiting the efflux function of multiple ATP-binding cassette transporters. Cancer Res. 2010;70 (20):7981-7991. doi:10.1158/0008-5472.CAN-10-0111

8. Burger RA, Brady MF, Bookman MA, et al. Incorporation of bevacizumab in the primary treatment of ovarian cancer. $N$ Engl $J$ Med. 2011;365(26):2473-2483. doi:10.1056/NEJMoa1104390

9. Jiang X, Tang H, Chen T. Epidemiology of gynecologic cancers in China. J Gynecol Oncol. 2018;29(1):e7. doi:10.3802/jgo.2018.29.e7

10. Chen W, Zheng R, Baade PD, et al. Cancer statistics in China, 2015. CA Cancer J Clin. 2016;66(2):115-132. doi:10.3322/caac.21338

11. Korkmaz T, Seber S, Basaran G. Review of the current role of targeted therapies as maintenance therapies in first and second line treatment of epithelial ovarian cancer; In the light of completed trials. Crit Rev Oncol Hematol. 2016;98:180-188. doi:10.1016/j.critrevonc.2015.10.006

12. Petrillo M, Pedone Anchora L, Tortorella L, et al. Secondary cytoreductive surgery in patients with isolated platinum-resistant recurrent ovarian cancer: a retrospective analysis. Gynecol Oncol. 2014;134 (2):257-261. doi:10.1016/j.ygyno.2014.05.029

13. Coleman RL, Spirtos NM, Enserro D, et al. Secondary surgical cytoreduction for recurrent ovarian cancer. N Engl J Med. 2019;381 (20):1929-1939. doi:10.1056/NEJMoa1902626

14. Pal T, Permuth-Wey J, Betts JA, et al. BRCA1 and BRCA2 mutations account for a large proportion of ovarian carcinoma cases. Cancer. 2005;104(12):2807-2816. doi:10.1002/cncr.21536

15. Antoniou A, Pharoah PD, Narod S, et al. Average risks of breast and ovarian cancer associated with BRCA1 or BRCA2 mutations detected in case Series unselected for family history: a combined analysis of 22 studies. Am J Hum Genet. 2003;72(5):1117-1130. doi: $10.1086 / 375033$

16. Neff RT, Senter L, Salani R. BRCA mutation in ovarian cancer: testing, implications and treatment considerations. Ther Adv Med Oncol. 2017;9(8):519-531. doi:10.1177/1758834017714993

17. Chirnomas D, Taniguchi T, de la Vega M, et al. Chemosensitization to cisplatin by inhibitors of the Fanconi anemia/BRCA pathway. Mol Cancer Ther. 2006;5(4):952-961. doi:10.1158/1535-7163.MCT-050493

18. Tan DS, Rothermundt C, Thomas K, et al. "BRCAness" syndrome in ovarian cancer: a case-control study describing the clinical features and outcome of patients with epithelial ovarian cancer associated with BRCA1 and BRCA2 mutations. J Clin Oncol. 2008;26 (34):5530-5536. doi:10.1200/JCO.2008.16.1703

19. Yang D, Khan S, Sun Y, et al. Association of BRCA1 and BRCA2 mutations with survival, chemotherapy sensitivity, and gene mutator phenotype in patients with ovarian cancer. JAMA. 2011;306 (14):1557-1565. doi:10.1001/jama.2011.1456

20. Alsop K, Fereday S, Meldrum C, et al. BRCA mutation frequency and patterns of treatment response in BRCA mutation-positive women with ovarian cancer: a report from the Australian Ovarian Cancer Study Group. J Clin Oncol. 2012;30(21):2654-2663. doi: $10.1200 / J C O .2011 .39 .8545$

21. Farmer H, McCabe N, Lord CJ, et al. Targeting the DNA repair defect in BRCA mutant cells as a therapeutic strategy. Nature. 2005;434(7035):917-921. doi:10.1038/nature03445

22. Fong PC, Boss DS, Yap TA, et al. Inhibition of poly(ADP-ribose) polymerase in tumors from BRCA mutation carriers. $N$ Engl J Med. 2009;361(2):123-134. doi:10.1056/NEJMoa0900212 
23. Jiang $X$, Li W, Li X, Bai H, Zhang Z. Current status and future prospects of PARP inhibitor clinical trials in ovarian cancer. Cancer Manag Res. 2019;11:4371-4390. doi:10.2147/CMAR.S200524

24. Cairns J, Fridley BL, Jenkins GD, Zhuang Y, Yu J, Wang L. Differential roles of ERRFI1 in EGFR and AKT pathway regulation affect cancer proliferation. EMBO Rep. 2018;19(3). doi:10.15252/ embr.201744767

25. Zhang X, Pickin KA, Bose R, Jura N, Cole PA, Kuriyan J. Inhibition of the EGF receptor by binding of MIG6 to an activating kinase domain interface. Nature. 2007;450(7170):741-744. doi:10.1038/ nature 05998

26. Rawluk J, Waller CF. Gefitinib. Recent Results Cancer Res. 2018;211:235-246.

27. Mok TS, Wu YL, Thongprasert S, et al. Gefitinib or carboplatin-paclitaxel in pulmonary adenocarcinoma. $N$ Engl J Med. 2009;361(10):947-957. doi:10.1056/NEJMoa0810699

28. Yang CH, Yu CJ, Shih JY, et al. Specific EGFR mutations predict treatment outcome of stage IIIB/IV patients with chemotherapy-naive non-small-cell lung cancer receiving first-line gefitinib monotherapy. J Clin Oncol. 2008;26(16):2745-2753. doi:10.1200/JCO.2007.15.6695

29. Chang X, Izumchenko E, Solis LM, et al. The relative expression of Mig6 and EGFR is associated with resistance to EGFR kinase inhibitors. PLoS One. 2013;8(7):e68966. doi:10.1371/journal.pone.0068966

30. Borad MJ, Champion MD, Egan JB, et al. Integrated genomic characterization reveals novel, therapeutically relevant drug targets in FGFR and EGFR pathways in sporadic intrahepatic cholangiocarcinoma. PLoS Genet. 2014;10(2):e1004135. doi:10.1371/journal.pgen.1004135

31. Kim KJ, Li B, Winer J, et al. Inhibition of vascular endothelial growth factor-induced angiogenesis suppresses tumour growth in vivo. Nature. 1993;362(6423):841-844. doi:10.1038/362841a0

32. Matulonis UA. Bevacizumab and its use in epithelial ovarian cancer. Future Oncol. 2011;7(3):365-379. doi:10.2217/fon.10.167

33. Ellis LM, Hicklin DJ. VEGF-targeted therapy: mechanisms of anti-tumour activity. Nat Rev Cancer. 2008;8(8):579-591. doi:10.1038/nrc2403

34. Jain RK. Normalization of tumor vasculature: an emerging concept in antiangiogenic therapy. Science. 2005;307(5706):58-62. doi:10.1126/ science. 1104819

35. Jin Y, Wei L, Jiang Q, et al. Comparison of efficacy and toxicity of bevacizumab, endostar and apatinib in transgenic and human lung cancer xenograft zebrafish model. Sci Rep. 2018;8(1):15837. doi:10.1038/s41598-018-34030-5

36. Jiang W, Xu J, Wang R, Wang T, Shu Y, Liu L. Merkel cell carcinoma of the thigh: case report and review of the literature. Onco Targets Ther. 2019;12:535-540. doi:10.2147/OTT.S182169

37. Tian S, Quan H, Xie C, et al. YN968D1 is a novel and selective inhibitor of vascular endothelial growth factor receptor-2 tyrosine kinase with potent activity in vitro and in vivo. Cancer Sci. 2011;102(7):1374-1380. doi:10.1111/j.1349-7006.2011.01939.x

38. Zhang Y, Chen Y, Zhang D, Wang L, Lu T, Jiao Y. Discovery of novel potent VEGFR-2 inhibitors exerting significant antiproliferative activity against cancer cell lines. J Med Chem. 2018;61 (1):140-157. doi:10.1021/acs.jmedchem.7b01091

39. Tong XZ, Wang F, Liang S, et al. Apatinib (YN968D1) enhances the efficacy of conventional chemotherapeutical drugs in side population cells and ABCB1-overexpressing leukemia cells. Biochem Pharmacol. 2012;83(5):586-597. doi:10.1016/j.bcp.2011.12.007
40. Zhang M, Tian Z, Sun Y. Successful treatment of ovarian cancer with apatinib combined with chemotherapy: a case report. Medicine. 2017;96(45):e8570. doi:10.1097/MD.0000000000008570

41. Miao M, Deng G, Luo S, et al. A Phase II study of apatinib in patients with recurrent epithelial ovarian cancer. Gynecol Oncol. 2018;148(2):286-290. doi:10.1016/j.ygyno.2017.12.013

42. Lan C-Y, Wang Y, Xiong Y, et al. Apatinib combined with oral etoposide in patients with platinum-resistant or platinum-refractory ovarian cancer (AEROC): a Phase 2, single-arm, prospective study. Lancet Oncol. 2018;19(9):1239-1246. doi:10.1016/S1470-2045(18) 30349-8

43. Li CM, Liu ZC, Bao YT, Sun XD, Wang LL. Extraordinary response of metastatic pancreatic cancer to apatinib after failed chemotherapy: a case report and literature review. World J Gastroenterol. 2017;23 (41):7478-7488. doi:10.3748/wjg.v23.i41.7478

44. Scott AJ, Messersmith WA, Jimeno A. Apatinib: a promising oral antiangiogenic agent in the treatment of multiple solid tumors. Drugs Today. 2015;51(4):223-229. doi:10.1358/dot.2015.51.4.2320599

45. Ling Y, Yang Y, Lu N, et al. Endostar, a novel recombinant human endostatin, exerts antiangiogenic effect via blocking VEGF-induced tyrosine phosphorylation of KDR/Flk-1 of endothelial cells. Biochem Biophys Res Commun. 2007;361(1):79-84. doi:10.1016/j.bbrc.2007. 06.155

46. Li K, Shi M, Qin S. Current status and study progress of recombinant human endostatin in cancer treatment. Oncol Ther. 2018;6(1):21-43. doi:10.1007/s40487-017-0055-1

47. Yu M, Han Y, Zhuo H, Zhang S. Endostar, a modified endostatin induces vascular normalization to improve chemotherapy efficacy through suppression of src signaling pathway. Cancer Biother Radiopharm. 2018;33(4):131-138. doi:10.1089/cbr.2017.2399

48. Wang J, Sun Y, Liu Y, et al. [Results of randomized, multicenter, double-blind Phase III trial of rh-endostatin (YH-16) in treatment of advanced non-small cell lung cancer patients]. Zhongguo Fei Ai Za Zhi. 2005;8(4):283-290. Chinese. doi:10.3779/j.issn.1009-3419.20 05.04.07

49. Ding Y, Wang Y, Cui J, Si T. Endostar blocks the metastasis, invasion and angiogenesis of ovarian cancer cells. Neoplasma. 2020;67 (3):595-603. doi:10.4149/neo 2020 190716N640

50. Wang B, Xu L, Li Q, et al. Endostar continuous versus intermittent intravenous infusion combined with chemotherapy for advanced NSCLC: a systematic review and meta-analysis including non-randomized studies. BMC Cancer. 2020;20(1):1021. doi:10.11 86/s12885-020-07527-4

51. Hansma AH, Broxterman HJ, van der Horst I, et al. Recombinant human endostatin administered as a 28-day continuous intravenous infusion, followed by daily subcutaneous injections: a Phase I and pharmacokinetic study in patients with advanced cancer. Ann Oncol. 2005;16(10):1695-1701. doi:10.1093/annonc/mdi318

52. Chen Z, Guo W, Cao J, et al. Endostar in combination with modified FOLFOX6 as an initial therapy in advanced colorectal cancer patients: a phase I clinical trial. Cancer Chemother Pharmacol. 2015;75(3):547-557. doi:10.1007/s00280-014-2656-9

53. Hu X, Zhang J, Xu B, et al. Multicenter phase II study of apatinib, a novel VEGFR inhibitor in heavily pretreated patients with metastatic triple-negative breast cancer. Int $J$ Cancer. 2014;135 (8):1961-1969. doi:10.1002/ijc.28829 


\section{Publish your work in this journal}

OncoTargets and Therapy is an international, peer-reviewed, open access journal focusing on the pathological basis of all cancers, potential targets for therapy and treatment protocols employed to improve the management of cancer patients. The journal also focuses on the impact of management programs and new therapeutic agents and protocols on patient perspectives such as quality of life, adherence and satisfaction. The manuscript management system is completely online and includes a very quick and fair peer-review system, which is all easy to use. Visit http://www.dovepress.com/ testimonials.php to read real quotes from published authors. 